\title{
Osteochondritis of Intermediate Cuneiform with Delayed Appearance Ossification Centre of Navicular Bone-A Rarity
}

\author{
${ }^{1}$ Archit Agarwal, ${ }^{2}$ Rajesh Lalchandani
}

\begin{abstract}
Osteochondritis of intermediate cuneiform is an unusual entity in itself but it is even rarer to find it with delayed appearance of navicular ossification centre. We report a case who presented with unilateral deformity of foot. Diagnosis was established with help of ski gram and magnetic resonance imaging (MRI) of foot. Patient was managed conservatively. Thus osteochondritis can have varied presentation and affects mostly male children. On extensive review of literature we could not come across any report of such case from Indian subcontinent.
\end{abstract}

Keywords: Cuneiform, Navicular, Osteochondritis, Ossification centre

How to cite this article: Agarwal A, Lalchandani R. Osteochondritis of Intermediate Cuneiform with Delayed Appearance Ossification Centre of Navicular Bone-A Rarity. J Foot Ankle Surg (Asia Pacific) 2018;5(2):77-79.

\section{Source of support: Nil}

Conflict of interest: None

\section{INTRODUCTION}

Osteochondritis has been described as a developmental derangement of normal bone growth of centre of ossification of any epiphysis. Some even describe it as aseptic ischemic necrosis. It is a self-limiting condition affecting mostly children. ${ }^{1}$ This condition can take place at more than 28 sites in the body involving primary and secondary ossification centers. ${ }^{2}$ This case of osteochondritis of intermediate cuneiform is being reported because of its rarity and its association with absence of bilateral navicular bone ossification centre which has not been reported before.

\section{CASE REPORT}

A 7 year and 1-month-old child presented to the outpatient clinic along with his father and complained of

\footnotetext{
${ }^{1}$ Senior Resident, ${ }^{2}$ Specialist

1,2Department of Orthopedics, Employees State Insurance Postgraduate Institue of Medical Sciences and Research and Model Hospital, New Delhi, India

Corresponding Author: Archit Agarwal, Senior Resident, Department of Orthopedics, Employees State Insurance Postgraduate Institue of Medical Sciences and Research and Model Hospital, New Delhi, India, e-mail: dr.architagarwal@ gmail.com
}

deformity of the left foot of the child. His both feet were normal at birth, and the deformity was noticed by parents only a year back. The deformity has slowly become more evident over the last one year. The child did not complain of any pain in the foot at any point in time. There was no history of trauma. The gait was normal and no difference was seen at a different speed of walking. On inspection of the left foot, the heel was in valgus (Fig. 1), medial arch appeared to have collapsed, the toes were splayed, and forefoot was in abduction due to which the lateral border appeared concave outwards (Figs 1 and 2). On physical examination, the deformity was correctable. No tenderness or signs of inflammation were present.



Fig. 1: Clinical photograph showing splaying of toes and abduction of forefoot

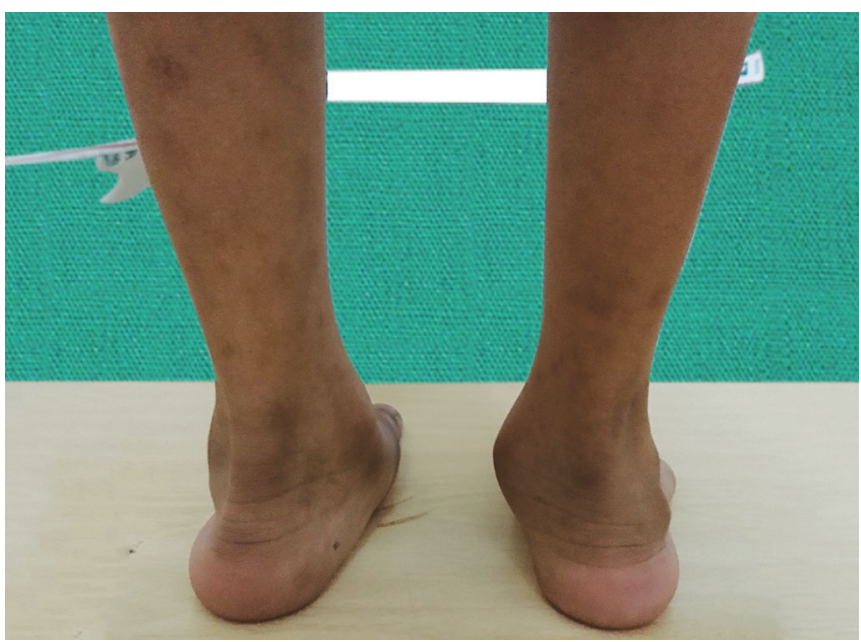

Fig. 2: Clinical photograph showing heel in valgus and collapsed medial arch 
The range of inversion-eversion at subtalar joint and plantarflexion dorsiflexion of the ankle was painless and full. On measurement, the left foot was $2 \mathrm{~cm}$ shorter in length in comparison to the other foot. Distal neurovascular status was normal.

Radiograph of the left foot (Fig. 3) showed sclerosed, irregular intermediate cuneiform which was smaller in size than the opposite side. In both feet, the navicular bone could not be visualized. On MRI, it was found that the left intermediate cuneiform ossific nucleus was small and irregular (Fig. 4). Ossification centre was not seen in bilateral navicular bone cartilage (Fig. 3). Lateral cuneiform is larger on the left than on the right foot. MRI revealed loss of signal intensity in intermediate cuneiform on the sagittal T1-weighted images. All the routine investigations and inflammatory and metabolic markers were within normal limits. There was no evidence of infective etiology.

The child was managed conservatively, and parents were reassured about the benign nature of the disease. Consent of the child's father was taken for publication of reports.

\section{DISCUSSION}

This report describes a rare case of idiopathic osteochondritis of intermediate cuneiform along with the delayed appearance of ossification centre of bilateral navicular bones. In the past, four such cases of osteochondritis of intermediate cuneiform have been reported, but this is unique because of its different clinical presentation from the other four reported cases in the past. This is the first Indian case to be recorded.

The foot is a common site of osteochondritis involving different bones in children. Osteochondritis of navicular bone has been named as Kohler's bone disease, the second metatarsal head as Freiberg's infraction, the base of 5th



Fig. 3: Radiograph of both feet showing small irregular intermediate cuneiform and absent navicular bone metatarsal as Iselin's disease, calcaneal apophysitis as Sever's disease and of medial cuneiform as Buschke's disease. Osteochondritis of medial cuneiform has been reported in literature more often than the intermediate cuneiform. ${ }^{3}$ In 1953 Hicks $^{2}$ reported the first case of osteochondritis of intermediate cuneiform. Since then just three more cases have been reported, the last one being in 2013. In all the cases, one thing was in commonpain in the foot. In contrast to all these reported cases, the child in this report never had pain and was brought to the hospital only because of the deformity of the foot.

In all the reported cases of osteochondritis of cuneiform bones, boys have been affected just as in this case. ${ }^{3}$ The theory behind this is that only those bones are affected whose ossification nucleus appears after birth. In boys, the ossification nucleus appears 8 months later than girls. The intermediate cuneiform and navicular are last to appear at the age of 4 . By this time the child starts walking, and the mechanical stress has increased. So, both developments of ossification nucleus and mechanical stress coincide more in boys than girls. ${ }^{4}$ The age group commonly affected is 4 to 6 years. By this time navicular as well as intermediate cuneiform ossification centre should have developed. But in the present case, navicular bone ossification has not started to appear even till 7 years of age and ossification centre of intermediate cuneiform is smaller and irregular due to osteochondritis. This has led to compensatory bony changes in the surrounding bones as seen in the MRI. In our case, the father described that the boy developed the deformity only since last one year. This means that the child could have developed osteochondrosis at the age of six.

Osteochondrosis of intermediate cuneiform has been described as benign and so underdiagnosed at times. Benign nature of its evolution and the short

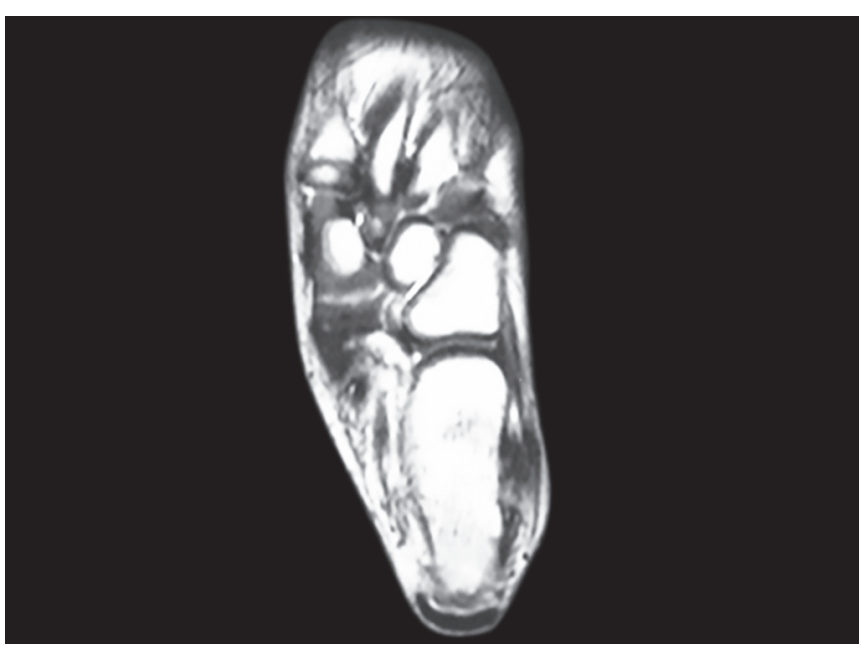

Fig. 4: MRI showing deformed ossific nucleus of intermediate cuneiform and absence of ossific nucleus in navicular bone cartilage 
duration of symptoms make it an underdiagnosed condition. It may be asymptomatic and therefore never diagnosed. Similar involvement of medial cuneiform has been reported to be diagnosed coincidently and the patient was asymptomatic. ${ }^{5}$ García-Mata ${ }^{3}$ advised to consider osteochondrosis as a physiological or nonpathological disorder or a variant of the normality, due to the short evolution of the process as in their case clinic-radiological features disappeared in few weeks. On the contrary, osteochondritis of intermediate cuneiform has been reported to produce antalgic gait, avoidance of weight bearing on the affected foot and even compensatory in-toeing in some cases. ${ }^{6}$

In the present case, it is interesting to note the absence of the ossification centre of the navicular bone in both feet. Normally, navicular bone and intermediate cuneiform are the last bone in the foot to begin ossification. In boys, it begins by 4 years but has been reported variably as between 2 to 6 years. ${ }^{5}$ Cases having osteochondritis of intermediate cuneiform and navicular bone simultaneously have been reported just three times previously. But in this case, osteochondritis of intermediate cuneiform is associated with the delayed appearance of navicular ossification centre. Osteochondritis of isolated navicular bone is far more common than other foot bones and is more often reported than that of intermediate cuneiforms. ${ }^{4}$

Exact etiology of osteochondritis is unknown. Different theories have been proposed-rapid growth, heredity, anatomical characteristics, repetitive microtrauma, vascular abnormalities, hormonal imbalances, and dietary factors. ${ }^{7}$ On carrying out differential diagnosis osteomyelitis needs to be ruled out. Eosinophilic granuloma and Ewing's sarcoma have also been considered in differential diagnosis in past. ${ }^{8}$ Especially in an endemic country like India tubercular osteomyelitis is a possibility because of its varied presentation.
Treatment is mainly conservative. Rest and symptomatic treatment with analgesics have been prescribed in symptomatic cases. Role of shoe inserts and casts has not been proved yet. Osteochondritis of intermediate cuneiform has been described as a self-limiting disorder which needs to be followed-up regularly and eventually it does not leave pathological sequelae. ${ }^{6}$ Even our patient had a similar pattern of progress from the time it was diagnosed till 6 months follow up. Invasive interventions like multiple drill holes to decompress the bone have been described to be more harmful than useful. ${ }^{6}$

\section{REFERENCES}

1. Resnick D. Diagnosis of bone and joint disorders. 4th Philadelphia, Pa: WB Saunders; 2002;4:3686-3742.

2. Hicks BTG. Case report. Osteochondritis of the tarsal second cuneiform bone. Br J Radiol 1953;26:214-215.

3. García-Mata S. Avascular necrosis of the intermediate cuneiform bone in a child: a very rare cause of limp in a child. A variant of the normality? Serafin Garc1 'a-Mata. Journal of Pediatric Orthopaedics B 2013;22:255-258.

4. Vaquero Martin J, Vicente-Herrera E, Pereiro de Lamo J, Vidal Fernandez C. Osteocondritis of the medial cuneiform. J Pediatr Orthop B 1999;8:69-71.

5. The juvenile skeleton Kose O, Demiralp B, Oto M, Sehirlioglu A. An unusual cause of foot pain in a child: osteochondrosis of the intermediate cuneiform. J Foot Ankle Surg 2009;48: 474-476.

6. Kose O, Demiralp B, Oto M, Sehirlioglu A. An unusual cause of foot pain in a child: osteochondrosis of the intermediate cuneiform. J Foot Ankle Surg 2009;48: 474-476.

7. Herring JA. The limping child. In: Herring JA, ed. Tachdjian's Pediatric Orthopaedics. Philadelphia, PA: W B Saunders; 2002:83-94.

8. Leeson MC, Weiner DS. Osteochondrosis of the tarsal cuneiforms. Clin Orthop 1985;196:260-264. 